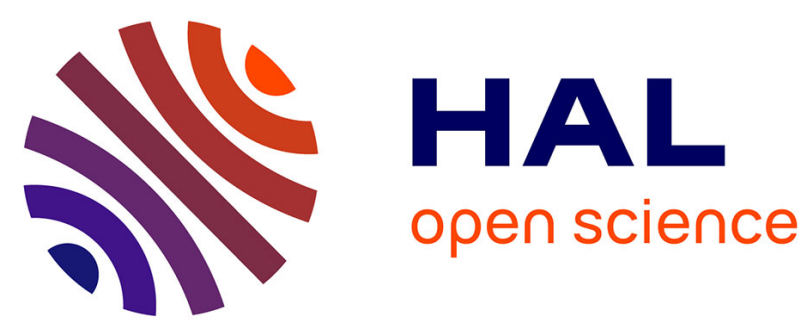

\title{
Unified microporomechanical approach for mechanical behavior and permeability of misaligned unidirectional fiber reinforcement
}

\author{
Thang Tran, Sébastien Comas-Cardona, Nor-Edine Abriak, Christophe \\ Binetruy
}

\section{To cite this version:}

Thang Tran, Sébastien Comas-Cardona, Nor-Edine Abriak, Christophe Binetruy. Unified microporomechanical approach for mechanical behavior and permeability of misaligned unidirectional fiber reinforcement. Composites Science and Technology, 2007, 10.1016/j.compscitech.2010.04.023 . hal00485514

\author{
HAL Id: hal-00485514 \\ https://hal.science/hal-00485514
}

Submitted on 21 May 2010

HAL is a multi-disciplinary open access archive for the deposit and dissemination of scientific research documents, whether they are published or not. The documents may come from teaching and research institutions in France or abroad, or from public or private research centers.
L'archive ouverte pluridisciplinaire HAL, est destinée au dépôt et à la diffusion de documents scientifiques de niveau recherche, publiés ou non, émanant des établissements d'enseignement et de recherche français ou étrangers, des laboratoires publics ou privés. 


\section{Accepted Manuscript}

Unified microporomechanical approach for mechanical behavior and permeability of misaligned unidirectional fiber reinforcement

Thang Tran, Sébastien Comas-Cardona, Nor-Edine Abriak, Christophe Binetruy

PII: S0266-3538(10)00168-5

DOI: 10.1016/j.compscitech.2010.04.023

Reference:

CSTE 4699

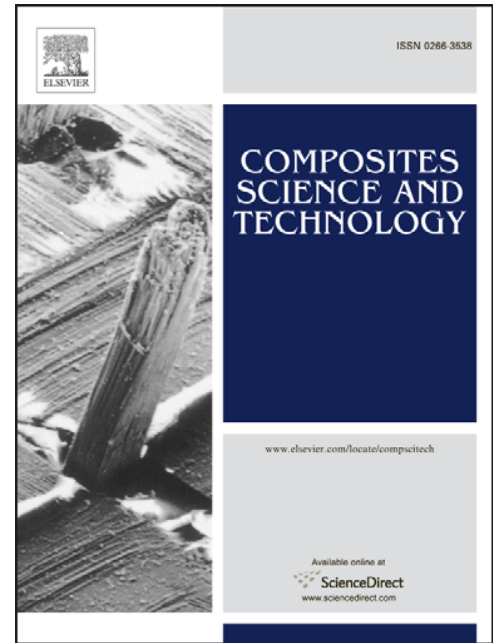

To appear in:

Composites Science and Technology

Received Date:

28 September 2009

Revised Date:

21 April 2010

Accepted Date:

23 April 2010

Please cite this article as: Tran, T., Comas-Cardona, S., Abriak, N-E., Binetruy, C., Unified microporomechanical approach for mechanical behavior and permeability of misaligned unidirectional fiber reinforcement, Composites Science and Technology (2010), doi: 10.1016/j.compscitech.2010.04.023

This is a PDF file of an unedited manuscript that has been accepted for publication. As a service to our customers we are providing this early version of the manuscript. The manuscript will undergo copyediting, typesetting, and review of the resulting proof before it is published in its final form. Please note that during the production process errors may be discovered which could affect the content, and all legal disclaimers that apply to the journal pertain. 


\title{
Unified microporomechanical approach for mechanical behavior and permeability of misaligned unidirectional fiber reinforcement
}

\author{
Thang Tran ${ }^{\mathrm{a}}$, Sébastien Comas-Cardona ${ }^{\mathrm{a}, *}$, \\ Nor-Edine Abriak ${ }^{\mathrm{b}}$, Christophe Binetruy ${ }^{\mathrm{a}}$ \\ ${ }^{a}$ École des Mines de Douai, Polymers and Composites Technology and Mechanical \\ Engineering Department, 941 rue Charles Bourseul, BP 10838, 59508 Douai \\ Cedex, France \\ ${ }^{\mathrm{b}}$ École des Mines de Douai, Civil and Environmental Engineering Department, \\ 941 rue Charles Bourseul, BP 10838, 59508 Douai Cedex, France
}

\begin{abstract}
The microporomechanical approach (via homogenization schemes) has been used and combined with triaxial tests to verify the Biot theory for the perfectly straight unidirectional fiber assembly in a previous paper [1]. The comparison of theoretical and experimental results is in good agreement, i.e. the Biot coefficients are clearly lower than one for densely packed fiber array. This result will be developed in this article in the case where the fibers are not perfectly straight but in misalignment (unidirectional fiber assembly in localized contact). Furthermore, within the same theoretical framework, the transverse compression modulus and the hydraulic permeability will be also estimated for the fiber reinforcement of double-scale porosity. The homogenization schemes used in this article are the self-consistent and the one proposed by Mori-Tanaka. The estimated and, when possible, bibliographical results for different types of fibrous materials (carbon, kevlar and glass fibers) are compared and show good agreement.
\end{abstract}

Key words: A Textile composites, B Mechanical properties, B Transport properties, Biot coefficients, Porous material

\footnotetext{
* Corresponding author. Tel.: +33 3277121 87; fax: +33 327712981 .

Email address: sebastien.comas@mines-douai.fr (Sébastien

Comas-Cardona).
} 


\section{Introduction}

Fiber reinforced organic composites are widely used for both low and high performance applications. Whatever the process used to obtain the final part, four major stages have to be achieved, optimized and controlled to obtain the highest part quality: lay-up, impregnation, consolidation and curing (for thermosetting resins) or cooling (for thermoplastic matrices) [2]. Failure in anyone of the four stages will lead to a non-optimal quality part that may have to be discarded because it does not fulfill the final mechanical requirements.

Helping manufacturers to produce high quality parts relies on models and simulation tools to prevent trial and error procedures and wasting time. Modeling and simulating manufacturing accurately requires a good understanding of the physics involved all along the process, such as fluid flow in fibrous media and hydro-mechanical coupling (during impregnation and consolidation), or the mechanical behavior of the fiber reinforcement for instance. Previous studies have focused on such modeling and simulations, but they are often based on very different theoretical approaches which make them difficult to combine and finding their parameters require experimental benches which are not always standardized. For example, dealing with injection of resin through a fibrous preform requires an appropriate knowledge of its permeability. On the other hand, the hydro-mechanical coupling is a relevant mechanism involved in processes where interaction between fluid and fiber occurs and can lead to driven or induced deformations of the fibrous medium.

Another specificity of the fiber reinforcements of concern in this study is that the porosity they exhibit is either single scale (unidirectional non-crimp fabrics NCF) or double scale (multidirectional NCF or woven fabrics). Fiber reinforcements, whether dry, partially saturated or fully saturated with a fluid, can be considered as engineered heterogeneous multiphase and multiscale materials. The most important heterogeneity of such a class of material is the porosity, i.e. the space in between the individual fibers at various scales ranging from inter-fiber volume at the microscopic scale to the interlayer space at the macroscopic scale. Porosity is the key parameter to understanding the fibrous reinforcement behaviour in its different fluid saturation states, in particular to predict transport properties as well as stiffness and deformation behaviours.

Because fibrous reinforcement are usually non periodic, fibrous reinforcement properties vary and some of them are difficult to tackle. This paper aims at evaluating within the framework of micromechanics of porous media, a unified continuum approach to simultaneously predict permeability and mechanical behavior of (fluid saturated or dry) preform and solid composite. Through suitable homogenization techniques, microscopic properties and behaviours of fibers and fluids are translated into macroscopic constitutive relations of 
mass transport phenomena and poro-mechanical deformation of fibrous media. First, a background on permeability and micromechanics is given. Then, theoretical developments of permeability and mechanical behavior of single and double scale fiber reinforcements are detailed and discussed before concluding.

\section{Background}

\subsection{Permeability}

The flow of a fluid through fabrics is generally described by a macroscopic theory of fluid flow known as Darcy's law [3,4]. It was originally proposed to describe the simple situation of a saturated flow in a rigid sand bed. Darcy's law is basically the macroscopic integration of the Navier-Stokes equations under certain conditions. That relation involves the permeability which represent the ability of the fluid to flow in the fibrous material. Saturated and unsaturated permeabilities can be of interest for composite manufacturing. The saturated permeability is measured once the fiber reinforcement is fully saturated whereas the unsaturated permeability is measured when the flow progresses through the reinforcement. Because the unsaturated property is only of interest in the flow front region during manufacturing, the present study focuses on the saturated permeability. Numerous publications have related results and measurement techniques. The first type of measurements are based on fluid injection experiments with:

- unidirectional flow and constant injection pressure $[5,6]$ or constant flow rate $[7,4]$;

- biaxial flow and constant injection pressure [8,9] or constant flow rate [10];

- through-thickness flow with constant pressure [11,5] or constant flow rate $[12,13]$

- three dimensional flows [14-16].

The proposed benches need specific equipment, e.g. pressure pots or pistons, to drive the fluid within the fiber reinforcement combined to a mold and appropriate instrumentation for adequate monitoring. Besides all the equipment previously cited, several errors can occur such as mold deflection, racetracking (edge effects) and capillary effects $[17,18]$.

The experimental sources of error lead to a scattering of the measurements, therefore some efforts have focused lately on replacing liquid by gas (air or nitrogen) $[19,20]$. The benches are quite similar to the ones using liquid, making sure the pressure transducers and flow rate sensors are appropriate. However, 
the data treatment is different since the main governing equations requires to take the gas compressibility into account. Some studies have presented permeability measurements, but very few have compared values obtained on both fluid injection and air flow benches.

A third way to measure permeability has been proposed and relies on the through-thickness compression of impregnated fiber reinforcements [21-23]. Those methods have the advantage to be continuous, that is to obtain permeability over a wide range of fiber volume fractions with a single fiber reinforcement sample. Results of permeabilities have been compared to the ones obtained on fluid injection benches and presented good agreements [23].

The fourth method under investigation is based on either analytical $[3,24,25]$ or numerical predictions solving Navier-Stokes equations. Usually, calculations are performed on cells (most of the time periodic) which represent the fiber reinforcement openness (inter tows or/and intra tows). Several method have been used: finite volume [26], finite element [27], lattice Boltzmann [28], meshless Lagrangian smoothed particle hydrodynamics [29] or the asymptotic homogenization [30] method. A limitation of numerical calculations is the difficulty to define a periodic unit cell that would be representative of the fiber reinforcement. Then, for methods requiring to solve equations on a very delimited domain, that unit cell has to be constructed in terms of geometry and mesh, which requires a particular effort.

Moreover, real impregnation processes may have a deformable fiber bed, and as a consequence its porosity and permeability vary along with the fluid progression. This underlines the need to use a consistent and unified framework to address coupled or uncoupled fluid and solid mechanics problems in order to simplify simulation tools. The next section focuses on the micromechanical derivation of macroscopic mechanical properties.

\subsection{Micromechanical approach}

Advanced structural polymer composites use fibers in high content. Fiber reinforcements are usually compacted to reach the targeted fiber volume fraction. The knowledge of the mechanical behavior of fiber reinforcements is fundamental during the processing of composites (RTM, VARTM, compression, autoclave consolidation...) because it influences the cycle time and the performances of the final composite part.

Fabric behaviour under transverse compression has been extensively studied both experimentally and theoretically. Most of the experimental methods are based on a curve fitting approach of a law between the applied stress and either thickness, porosity or fiber volume fraction [31,32]. These simple mod- 
els contain two or more empirical constants that are difficult to correlate to fabric microscale or macroscale geometry. Those models are then of limited interest with the aim to relate mechanical properties of the fiber preform to microstructure. Micromechanical models based on beam theory have been proposed to overcome the above-mentioned drawbacks [33]. While they may provide a fairly good description of the stress/strain behaviour of dry fabrics, they cannot easily address the fluid/solid interaction at a microscale. Microporomechanics offer a consistent framework to deal with this question, to predict the effective homogenized mechanical properties of the fiber medium knowing micro-structural parameters and the mechanical properties of fibers.

To have an accurate law for mechanical and hydromechanical properties, it is necessary to determine the fourth-order homogeneous stiffness tensor $\mathbb{C}^{\text {hom }}$ and the Biot tensor $\boldsymbol{B}$ of the fiber assembly. The Biot tensor $\boldsymbol{B}$, once again, is determined as a function of $\mathbb{C}^{\text {hom }}$, so the difficulty of the mechanical behavior law is the determination of $\mathbb{C}^{\text {hom }}$ tensor. More details on that matter have been given in [1].

Once the $\mathbb{C}^{\text {hom }}$ tensor is determined, the transverse compression modulus of assembly $E_{1}$ is deduced. In most cases of composites processing, the compression of the fiber reinforcement is unidirectional, according to the transverse direction, thus the transverse compression modulus $E_{1}$ is important and need to be determined.

Estimation of the mechanical behavior and the permeability of a commercial reinforcement requires to improve the previous work [1] with features such as unidirectional fibers with misalignment (localized contacts) and doublescale porosity. The Mori-Tanaka estimate shows a good applicability for the calculation of stiffness and of permeability tensors ( $\mathbb{C}^{\text {hom }}$ and $\boldsymbol{K}$, respectively) of the fiber reinforcement of double-scale porosity.

\section{Microporomechanical approach for misaligned unidirectional as- semblies of single-scale porosity}

In the previous work [1], the bundles have been idealized with perfectly straight fibers. In reality, the bundle can be represented by a unidirectional fiber assembly of single-scale porosity containing several families of pores (with various shapes and a unique characteristic length). In that case, the geometrical distribution of unidirectional fibers in a bundle have to be known prior to any calculation of the transverse compression modulus or permeabilities. 


\subsection{Geometrical distribution of unidirectional fibers in a bundle}

The microstructural analysis of a bundle can be a complex and time consuming task. Several authors have studied unidirectional assemblies using point process [34-36]. Although a point is considered dimensionless, real transverse sections of fiber are represented as points when distances between them are rather important and their physical size is irrelevant. Looking at bundles, the distribution of fibers in their transverse section is not periodic. The hypothesis of complete randomness (the location of each point is independent of the location of any other points) is the simplest and the most realistic representation $[25]$.

Various studies have been contributing to determine the distribution of fibers in the transverse section of a bundle, e.g. Dirichlet tessellation (or Voronoi polygon) [37], fractal concept [38], intensity function, pair correlation function or mark correlation function $[35,36]$ but none of them studied the neighboring fiber distances in the transverse section of a bundle. A neighboring fiber is one that shares a side of the Voronoi polygon with the fiber interest (Fig. 1).

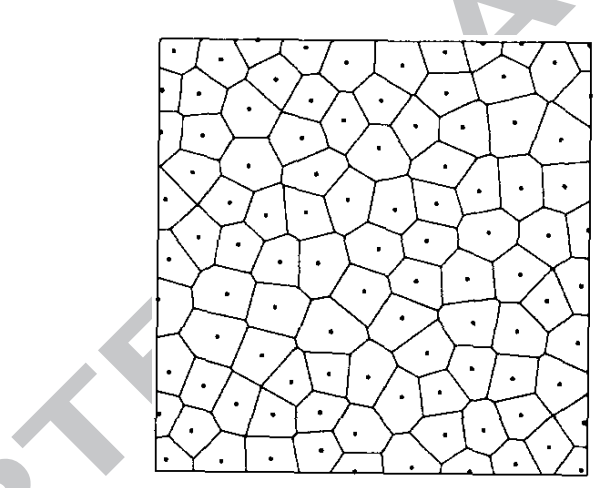

Fig. 1. Fiber distribution in the transverse section of bundle and the corresponding Voronoi polygons [35]

Based on the asymmetry of the experimental probability density distribution of neighboring fiber distance $[39,35,40]$, the Gamma probability density function $f\left(x_{1} ; a, k, \theta\right)$ is proposed for the theoretical approach and depends on the neighboring fiber distance $x_{1}$ and three parameters $a, k, \theta$

$$
f\left(x_{1} ; a, k, \theta\right)=\frac{1}{\theta \Gamma(k)}\left(\frac{x_{1}-a}{\theta}\right)^{k-1} \exp \left[-\left(\frac{x_{1}-a}{\theta}\right)\right] \quad x_{1} \in[a ;+\infty)
$$

where $\Gamma$ is the Gamma function. The parameters $a, k, \theta$ are determined as function of the fiber diameter $d$ and bundle volume fraction $V_{f}$ by the analysis of the microstructure of the unidirectional fibers in a bundle: 
- the smallest distance between two any fiber centers is $d$, thus $a=d$;

- the mean value of the Gamma probability density function $k \theta$ is equal to the hexagonal array distance of the equi-diameter fiber $l_{1}$ minus the diameter $d$

$$
k \theta=l_{1}-d=d\left[\sqrt{\frac{\pi}{2 \sqrt{3} V_{f}}}-1\right]
$$

- the analysis of the distribution feature of the fibers in a bundle gives

$$
k=\frac{1}{V_{f}^{\alpha}}
$$

where $\alpha$ is a parameter.

The Gamma function parameters $a, k, \theta$ are adjusted to the semi-empirical results of Pyrz [35]. The distance distribution of the unidirectional fiber in the transversal section of bundle follow the Gamma distribution (Eq. 1) whose parameters are given in Eq. 4.

$$
a=d ; \quad k=\frac{1}{\sqrt{V_{f}}} ; \quad \theta=d\left(0.95-\sqrt{V_{f}}\right)
$$

The point process was helpful in determining the inter-fiber distance, but it cannot be used for the determination of the distance between two contacts according to the longitudinal direction of the assembly. At low fiber volume fraction, the fiber contact can be punctual, at higher fiber volume fraction, the contact can be a line according to the longitudinal direction of the fiber. To determine the average distance between two contacts, let assume that the ratio $\omega$ of the distance between two longitudinal contacts $x_{3}$ over the distance between two edges of the neighboring fibers $x_{1}-d$ is constant. Thus when the unidirectional assembly is transversely compressed, the inter-fiber distance reduces, while the contact line distance increases and the distance between two longitudinal contacts decreases

$$
\omega=\frac{x_{3}}{x_{1}-d}=\text { constant }
$$

The ratio $\omega$ is determined by exploiting the experimental bibliographical results of Yurgartis [41] and Lee et al. [42]. In these works, authors measured the misalignment angles of unidirectional assembly of carbon fiber and represented them as probability density curves. The mean misalignment angles have been found equal to $1.936^{\circ}$ and $1.9^{\circ}$, respectively (the mean misalignment angle of the $8 \mathrm{~mm}$-thick laminate is chosen in the work of Lee et al. to avoid compression edge effects). Thus, the mean misalignment $\varphi$ is chosen in this work as $1.9^{\circ}$. 


$$
\omega=\frac{l_{3}}{l_{1}-d}=\frac{a_{3}}{a_{1}}=\operatorname{cotan} \varphi=30
$$

where $l_{1}$ and $l_{3}$ are mean values of $x_{1}$ and $x_{3}$, respectively; $a_{1}$ and $a_{3}$ are the semi-axes of the ellipsoidal porosity (Fig. 2). The mean inter-fiber distance $l_{1}$ and between two longitudinal contacts $l_{3}$ (as well as the ratio $\omega$ ) will help to the determination of the ellipsoidal pore size generated by the misalignment of fibers.

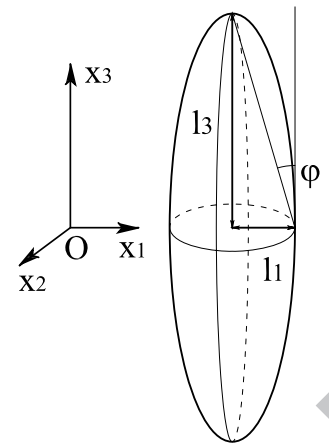

Fig. 2. Ellipsoidal porosity corresponding to the localized contact

\subsection{Micromechanical approach}

In this section, the Biot coefficients and the permeability will be estimated by the Mori-Tanaka and self-consistent schemes for the bundle of single-porosity. Following the assumptions of these schemes, the real fiber assembly is modelled by a reference medium which contains ellipsoidal inclusions. The reference medium can be chosen as the fiber or the homogenized material, depending on the schemes. According to the ratio of major axis to minor axis, the inclusion can be a cylinder, a sphere or an ellipsoid. The porous inclusions for the calculation of the assembly of single-scale porosity are cylindrical and ellipsoidal. Such approach do not take into consideration eventual sliding or rotations of the constituents of the porous material. Therefore the validity of the modeling and the predictions are limited to high volume fractions of constituents where sliding and rotations are greatly reduced due to high packing. Also note that in micromechanics, the results are usually plotted along the full range of porosity even if the validity of the models are less extended. Readers should focus on the high levels of volume fractions or low porosity ranges.

The volume fraction of the cylindrical pore is constant $\left(c_{c}\right)$ and represents the minimum porosity of the compact hexagonal fiber array. The equivalent radius $R_{c}$ is obtained by identifying the area of the real transverse and cylindrical sections 


$$
R_{c}=R \sqrt{\frac{2 \sqrt{3}-\pi}{2 \pi}}
$$

where $R$ is the radius of an equi-diameter fiber.

The volume fraction of the ellipsoidal pore $c_{e}$ is the remaining porosity

$$
c_{e}=c-c_{c}
$$

where $c$ is the total porosity of the assembly.

The minor semi-axis of the ellipsoid $a_{1}$ is $a_{1}=\left(l_{1}-d\right) / 2$, the major semi-axis is $a_{3}=l_{3} / 2$. The semi-axes $a_{1}$ and $a_{3}$ are used to compute the Biot coefficients and permeability estimates of unidirectional assemblies of single-scale porosity.

\subsubsection{Biot coefficients estimate}

The Biot coefficients will be estimated by the Mori-Tanaka scheme, which has proved more advantages compared to the dilute and Ponte Castañeda-Willis schemes for the calculation of fiber assembly [1]. The calculation assumptions for the unidirectional fiber assembly in localized contacts are:

- the fiber and the fiber assembly are both linear elastic;

- the real fiber assembly (Fig. 3) is modelled by a reference medium, the fiber, which contains the cylindrical and ellipsoidal pores (Fig. 4).

The ellipsoidal pore is illustrated as a "closed" pore in Fig. 4, but it implicitly characterizes an "opened" pore because its rigidity is null.

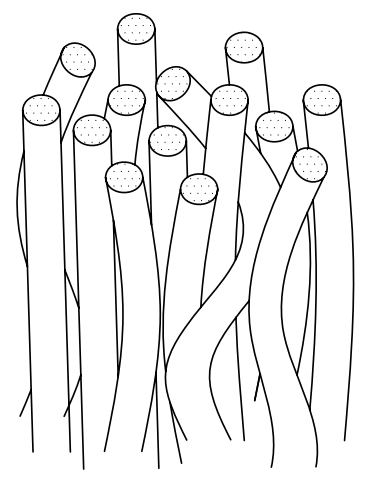

Fig. 3. Real unidirectional fiber assembly with misalignment

The Biot coefficients are determined by the following formulae [43] 


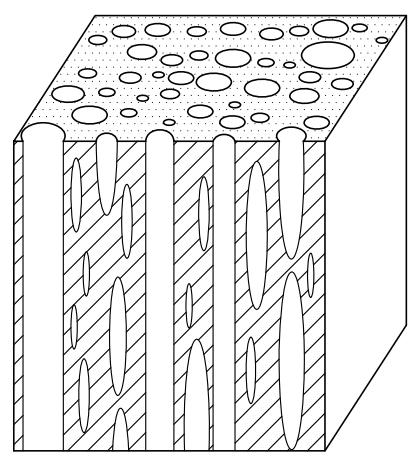

Fig. 4. Modelled unidirectional fiber assembly with misalignment

$$
\boldsymbol{B}=\boldsymbol{\delta}\left(\mathbb{I}-\mathbb{S}_{f}: \mathbb{C}_{M T}^{h o m}\right)
$$

where $\boldsymbol{\delta}$ is the second-order unit tensor; $\mathbb{I}$ is the fourth-order unit tensor; $\mathbb{S}_{f}$ is the compliance tensor of the fiber and $\mathbb{C}^{\text {hom }}$ is the homogeneous stiffness tensor. The latter is calculated by the Mori-Tanaka scheme

$$
\mathbb{C}_{M T}^{h o m}=(1-c) \mathbb{C}_{f}:\left((1-c) \mathbb{I}+\sum_{i} c_{i}\left(\mathbb{I}-\mathbb{S}_{i}^{E}\right)^{-1}\right)^{-1}
$$

where $\mathbb{C}_{f}$ is the stiffness tensor of the fiber; $\mathbb{S}^{E}$ is the Eshelby tensor. The index $i$ refers to $c$ (cylindrical pore) or $e$ (ellipsoidal pore).

Because of the presence of ellipsoidal pores, the Eshelby tensor cannot be determined analytically [44], thus the homogeneous stiffness tensor $\mathbb{C}_{M T}^{\text {hom }}$ is calculated numerically within the Walpole basis [45].

Figure 5 shows the Biot coefficient curves of the assembly of fibers of Kevlar 29 and carbon at ratios of major to minor axes of the ellipsoidal pore $\omega=30$. Note that both fibers are transversely isotropic. The curves of fibers of Kevlar 29 are compared to those of perfectly straight unidirectional fiber assembly ones $(\omega=+\infty)$. The Biot coefficients of perfectly straight assembly of transversely isotropic fibers can be calculated analytically because there are only cylindrical pores.

$$
\begin{gathered}
b_{1}=b_{2}=c \frac{2 c\left(E_{3}-E_{1} \nu_{31}^{2}\right)}{E_{3}\left[c\left(1+\nu_{12}\right)+\left(1-\nu_{12}\right)\right]-2 E_{1} \nu_{31}^{2}} \\
b_{3}=\frac{E_{3}\left\{\left(1+\nu_{12}\right)\left[2 \nu_{31}(1-c)+c\right]+\left(1-\nu_{12}\right)\right\}-2 E_{1} \nu_{31}^{2}}{E_{3}\left[c\left(1+\nu_{12}\right)+\left(1-\nu_{12}\right)\right]-2 E_{1} \nu_{31}^{2}}
\end{gathered}
$$


where $E_{i}$ are the elastic moduli according to the direction $i ; \nu_{i j}$ are the Poisson ratios in the plane $i j$. Here the direction $O x_{3}$ is oriented along the fiber axis and the $\left(O x_{1} ; O x_{2}\right)$ plane is the transverse one.

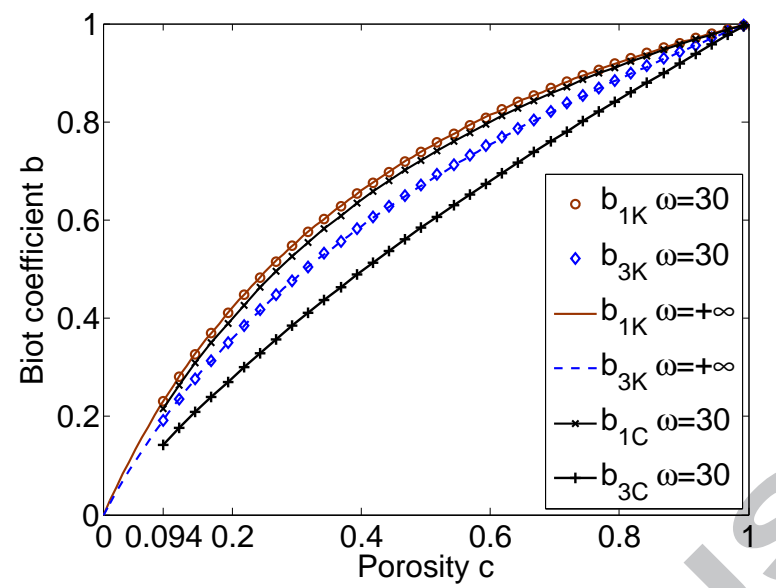

Fig. 5. Biot coefficients for unidirectional fiber assembly in localized $(\omega=30)$ and in perfect $(\omega=+\infty)$ contacts. The mechanical characteristics of the fiber of Kevlar 29 (subscript "K") are: $E_{3}=72 \mathrm{GPa} ; E_{1}=5.5 \mathrm{GPa} ; \mu_{31}=2 \mathrm{GPa}$ (axial shear modulus); $\nu_{31}=0.36 ; \nu_{12}=0.3$ [46]; of the fiber of carbon (subscript "C") are: $E_{3}=220 \mathrm{GPa} ; E_{1}=14 \mathrm{GPa} ; \mu_{31}=9 \mathrm{GPa} ; \nu_{31}=0.2 ; \nu_{12}=0.25[47]$

The comparison of the Biot coefficient curves in Fig. 5 leads to the following conclusions:

- the ellipsoidal form of pore (via $\omega$ ratio) seems to less influence the Biot coefficients. The Biot coefficients for unidirectional fiber assembly in localized and in perfect contacts coincide. More precisely, $b_{1(\omega=+\infty)}>b_{1(\omega=30)}$ and $b_{3(\omega=+\infty)}<b_{3(\omega=30)}$ for a very small value. That means, the rigidity according to the axis direction of the cylindrical pore is highest, and smallest for the transverse directions. These rigidities reduce in the longitudinal and increase in the transverse directions if the pore is ellipsoidal, and equal if the pore is spherical. The difference between the Biot coefficients for unidirectional fiber assembly in localized and in perfect contacts becomes significant when $\omega<5$;

the Biot coefficients for a perfectly straight unidirectional fiber assembly is independent of the axial shear modulus of the fiber $\mu_{31}$ (Eqs. 11 and 12), but it is not the case of unidirectional fiber assembly in localized contact because the presence of ellipsoidal pore causes the sliding during the isotropic compression. However the axial shear modulus slightly influences the Biot coefficients;

- the Biot coefficient curves of unidirectional fiber assembly in localized contact start at a porosity of 0.094 because it represents the minimal porosity of a unidirectional equi-diameter fiber assembly;

- the Biot coefficients are lower than one because of the low Poisson ratios of aramid fiber (same trend for carbon fiber). 


\subsubsection{Permeability tensor estimate}

The homogenization method is the calculation of macroscopic characteristic of material as function of constituent characteristics and microstructure. For the case of assembly of compact fiber, the macroscopic permeability cannot be calculated as function of the fiber permeability because the latter is null and therefore does not allow any flow. For this reason, the reference medium cannot be chosen as the fiber, thus the Mori-Tanaka scheme is no longer applicable. The only scheme that can be used in this case is the self-consistent where the reference medium is the homogenized one.

The unidirectional fiber assembly in localized contact (Fig. 3) is modelled by a homogeneous medium which contains three types of inclusions: cylindrical pore, ellipsoidal pore and cylindrical fiber (Fig. 6).

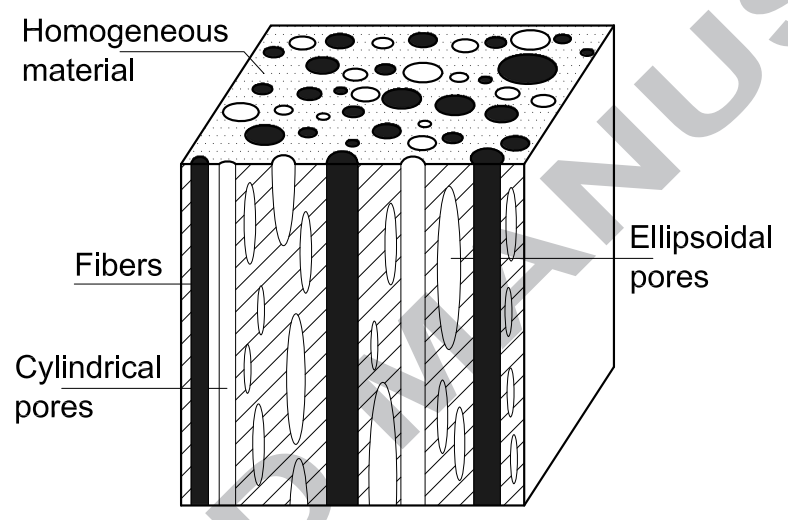

Fig. 6. Modelled material for the permeability determination by the self-consistent scheme

The homogeneous permeability tensor of the self-consistent scheme $\boldsymbol{K}_{S C}^{\text {hom }}$ writes [43]

$$
\boldsymbol{K}_{S C}^{h o m}=\left\langle\boldsymbol{K}_{i}\left[\boldsymbol{I}+\boldsymbol{P}_{i S C}^{h o m}\left(\boldsymbol{K}_{i}-\boldsymbol{K}_{S C}^{h o m}\right)\right]^{-1}\right\rangle\left\langle\left[\boldsymbol{I}+\boldsymbol{P}_{i S C}^{h o m}\left(\boldsymbol{K}_{i}-\boldsymbol{K}_{S C}^{h o m}\right)\right]^{-1}\right\rangle^{-1}
$$

where $\boldsymbol{K}_{i}$ is the permeability tensor of the inclusion $i$; $\boldsymbol{I}$ is there second-order unit tensor and $\boldsymbol{P}_{i S C}^{h o m}$ is a tensor calculated by

$$
\boldsymbol{P}_{i S C}^{h o m}=\boldsymbol{S}_{i}^{E}\left(\boldsymbol{K}_{S C}^{h o m}\right)^{-1}
$$

where $\boldsymbol{S}_{i}^{E}$ is the Eshelby tensor for the permeability calculation [44].

As already mentioned, the permeability of the fiber is null. The permeability tensor of the cylindrical pore is calculated from the Poiseuille flow solution in 
a tube of radius $R_{c}$

$$
\boldsymbol{K}_{c}=\left(\begin{array}{ccc}
0 & 0 & 0 \\
0 & 0 & 0 \\
0 & 0 & \frac{R_{c}^{2}}{8}
\end{array}\right)
$$

The permeability tensor of the ellipsoidal pore is

$$
\boldsymbol{K}_{e}=\left(\begin{array}{ccc}
K_{e 1} & 0 & 0 \\
0 & K_{e 2} & 0 \\
0 & 0 & K_{e 3}
\end{array}\right)
$$

where $K_{e i}$ is the permeability according to the direction $i$, they are calculated from the flow solution in a tube of elliptical section (whose semi-axes are $a_{e j}$, $\left.a_{e k}\right)$. For example, the permeability according to the direction $O x_{1}$ is [48]

$$
K_{e 1}=\frac{a_{e 2}^{2} a_{e 3}^{2}}{4\left(a_{e 2}^{2}+a_{e 3}^{2}\right)}
$$

The flow through the ellipsoidal pore is assimilated to the flow in a tube of ellipsoidal section. The relative contribution of the permeabilities in Eq. 13 is dictated by the Eshelby tensor $\boldsymbol{S}_{i}^{E}$.

Equation 13 is calculated numerically and iteratively by giving an initial guess of the homogeneous permeability tensor $\boldsymbol{K}_{S C}^{t}$ ( $t$ is the iteration counter)

$$
\boldsymbol{K}_{S C}^{t+1}=\left\langle\boldsymbol{K}_{i}\left[\boldsymbol{I}+\boldsymbol{P}_{S C}^{t}\left(\boldsymbol{K}_{i}-\boldsymbol{K}_{S C}^{t}\right)\right]^{-1}\right\rangle\left\langle\left[\boldsymbol{I}+\boldsymbol{P}_{S C}^{t}\left(\boldsymbol{K}_{i}-\boldsymbol{K}_{S C}^{t}\right)\right]^{-1}\right\rangle^{-1}
$$

The iterative resolution (Eq. 18) is stopped when

$$
\left|\frac{K_{p q, S C}^{t+1}-K_{p q, S C}^{t}}{K_{p q, S C}^{t}}\right|<\varepsilon_{e r}
$$

where the subscript $p q$ are the components of the permeability tensor; $\varepsilon_{e r}$ is the maximum allowed relative error. 
The calculation of the permeability tensor $\boldsymbol{K}_{S C}^{\text {hom }}$ is stopped when $\varepsilon_{e r}=10^{-6}$. The predictions of $\boldsymbol{K}_{S C}^{\text {hom }}\left(\mathrm{m}^{2}\right)$ are presented in Fig. 7 as a function of the bundle porosity $c$. They are compared to the Gebart's model (Eqs. 20 and 21) [49], which are used for the unidirectional fiber assembly in non compact hexagonal array

$$
K_{G 1}=K_{G 2}=\frac{16}{9 \pi \sqrt{2}}\left(\sqrt{\frac{1-c_{c}}{1-c}}-1\right)^{5 / 2} R^{2}
$$

$$
K_{G 3}=\frac{8}{57} \frac{c^{3}}{(1-c)^{2}} R^{2}
$$

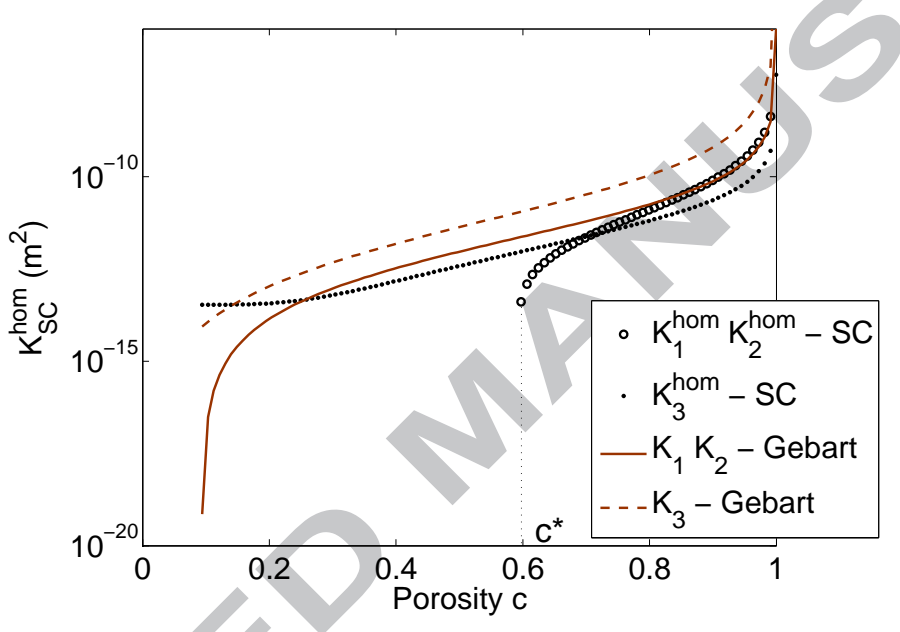

Fig. 7. The permeability of unidirectional fiber assembly calculated by the self-consistent (SC) scheme and Gebart formulae [49]. The diameter of the fiber is $15 \mu \mathrm{m}$ (corresponding to glass fibers)

The permeability $K_{3 S C}^{h o m}$ exists above $c_{c}$ (porosity of the cylindrical pore, which corresponds to the porosity of the compact hexagonal fiber array) because there is always a minimum (non zero) porosity according to this direction. On the other hand, the permeabilities in the transverse directions of assembly $K_{1 S C}^{h o m}$ and $K_{2 S C}^{h o m}$ are null for a porosity smaller than $c^{*} \approx 0.6$. Beyond this threshold, the existence of the macroscopic permeability is interpreted as the result of the pore connectivity, especially of ellipsoidal pores. The selfconsistent scheme thus takes into account the notion of percolation threshold [50]. The connectivity of pores results from the porosity increase in the modeled material.

In comparison to the permeability curves of a unidirectional fiber assembly in non compact hexagonal array predicted by the Gebart's model [49], the permeabilities calculated by the self-consistent scheme are lower. This was expected because Gebart's model assumes perfectly aligned fibers, which is not the case when misalignment is introduced in the bundle. Moreover, according 
to the Gebart's model, the longitudinal permeability is always higher than the transverse ones, while for the self-consistent scheme, this is only true for a porosity lower than $c \approx 0.7$.

The calculation by the self-consistent scheme allows to establish the following conclusions for the permeability tensor calculation of unidirectional fiber assembly of single-scale porosity by micromechanical approach:

- the self-consistent scheme can be used for the permeability tensor calculation of unidirectional fiber assembly of single-scale porosity. This is due to a representation of the reference medium which is homogeneous (because any component of material can be chosen as the reference medium);

- the application of the self-consistent scheme is very limited because the permeability is null for a porosity smaller than a critical one $c^{*}$ (the threshold of pore connectivity). The threshold depends on the shape of the considered pore. For the assumption of the cylindrical and ellipsoidal pores corresponding to the considered case, $c^{*}$ is high $(\approx 0.6)$, thus the existing interval of the permeability tensor is not interesting because it corresponds to the composites processing at the end of the consolidation;

- the self-consistent scheme equation is solved iteratively and numerically (except for some particular cases of inclusions);

- for high porosity, the model can be questionable since the flow is not dominated anymore by flow in channels (Eq. 17).

Thus, these remarks suggest that the micromechanical approach does not well adapt to the permeability tensor calculation of the unidirectional fiber assembly of single-scale porosity. Therefore for the the single-scale porosity medium, models based on the lubrication approaches are used instead of the micromechanical ones in the following.

\section{Microporomechanical approach for unidirectional reinforcement of double-scale porosity}

In reality, most of fiber reinforcements contain double-scale porosities (e.g. multiaxial non-crimp fabric, woven fabric... ) For these reinforcements, micropores and macropores exist. The micropores are located in the bundle (intrabundle pores) which have already been studied in section 3. The macropores are surrounding the bundles (inter-bundle or inter-layer pores) and are caused by the bundles arrangement within a ply and by ply lay-up.

This section studies the compression modulus and the permeability of the unidirectional non-crimp fabric. It consists of unidirectional fiber bundles, which are assembled by small transverse and longitudinal threads. The bundle itself 
consists of unidirectional fibers in localized contact.

\subsection{Transverse compression modulus}

The compression modulus is calculated for a non-crimp unidirectional reinforcement made of high strength carbon fiber (Fig. 8). For this reinforcement, there are two types of pores: micropore (intra-bundle) and macropore (interbundle and inter-layer). Very fine longitudinal and transverse threads hold the bundle (Fig. 9). The thickness of these threads (according to the direction $\mathrm{Ox}_{2}$ ) is very small. Moreover, the compression modulus is calculated only at a high compressive force leading to a small porosity in the reinforcement, which is a condition of validity for the estimated schemes. That is why the inter-layer macropore (a) is neglected and only the inter-bundle (ellipsoidal) pore (b) is considered. The properties of the inter-bundle pore are given in Tab. 1. These properties are obtained by image analysis where $a_{1}, a_{2}$ and $a_{3}$ are the three semi-axes of the modelled ellipsoidal pore according to the corresponding directions. During the compression, the thickness of reinforcement reduces, but it is assumed that the distances between the bundles and between the transverse threads remain unchanged. Therefore, values of $a_{1}$ and $a_{3}$ are supposed constant.

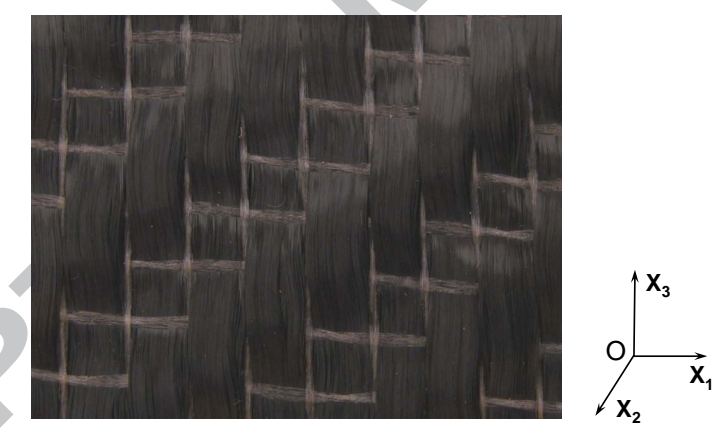

Fig. 8. Non-crimp unidirectional fabric

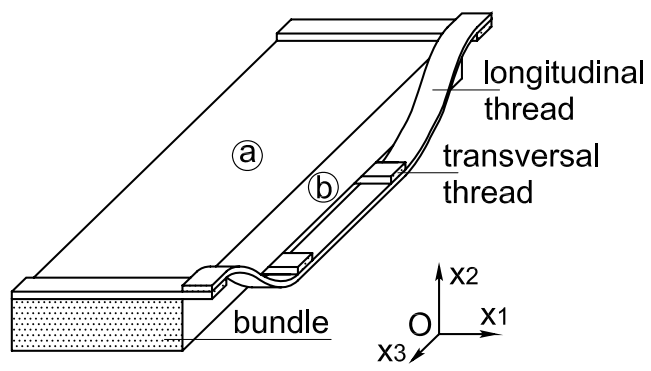

Fig. 9. Bundle with very fine longitudinal and transverse threads

Since the non-crimp UD reinforcement is a double-scale porosity medium, a double homogenization is required to assess macroscopic properties. The first homogenization is calculated similarly to that of the compression modulus of 
Table 1

Ellipsoidal macropore properties

\begin{tabular}{|l|c|}
\hline & Ellipsoidal macropore \\
\hline Volume fraction & 0.12 \\
\hline Semi-axis $a_{1}(\mathrm{~mm})$ & 0.5 \\
\hline Semi-axis $a_{2}(\mathrm{~mm})$ & $h / 2($ Eq. 23$)$ \\
\hline Semi-axis $a_{3}(\mathrm{~mm})$ & 6 \\
\hline
\end{tabular}

the bundle as function of the fiber properties and the bundle microstructure (fiber volume fraction caused by the fiber misalignment). This homogenization step has already been carried out in section 3.2.1. The second homogenization consists in the calculation of the compression modulus of the non-crimp UD reinforcement as function of the bundle modulus and the reinforcement microstructure (caused by the bundle arrangement). The real reinforcement is modelled by a reference medium (the bundle) which contains ellipsoidal macropores. The Mori-Tanaka formula is used for the double-scale porosity (Eq. 10).

Owing to the presence of ellipsoidal macropore of three different semi-axes, the non-crimp UD fabric is no longer transversely isotropic but orthotropic, thus the homogeneous stiffness tensor is composed of 9 independent components. The micromechanical calculation is carried out within the Walpole basis for orthotropic material [45]. For example, the compliance tensor $\mathbb{S}$ of the orthotropic material writes in the Walpole basis

$$
\mathbb{S}=\left\{\left[\begin{array}{ccc}
\frac{1}{E_{1}} & \frac{-\nu_{21}}{E_{2}} & \frac{-\nu_{31}}{E_{3}} \\
\frac{-\nu_{21}}{E_{2}} & \frac{1}{E_{2}} & \frac{-\nu_{32}}{E_{3}} \\
\frac{-\nu_{31}}{E_{3}} & \frac{-\nu_{32}}{E_{3}} & \frac{1}{E_{3}}
\end{array}\right], \frac{1}{2 \mu_{12}}, \frac{1}{2 \mu_{23}}, \frac{1}{2 \mu_{13}}\right\}
$$

The compression modulus $E_{2}$ according to the direction $O x_{2}$ is estimated numerically in the fiber content interval $[0.65 ; 0.75]$ because this is an interesting interval of porosity of the material during composite manufacturing and it complies with the values given in Tab. 1 and the assumption that the pore (a) (Fig. 9) does not exist. The thickness of the layer (axis $a_{2}$ ) is deduced from the fiber volume fraction $V_{f}$

$$
h=\frac{\rho_{s}}{V_{f} \rho_{v}}
$$


where $\rho_{s}$ is the areal weight of the non-crimp UD reinforcement and $\rho_{v}$ is the density of the fiber. This extrapolation is valid only for a small interval of $V_{f}$.

The estimated modulus $E_{2 M T}^{\text {hom }}$ of the non-crimp UD reinforcement is presented in Fig. 10. The compression modulus is smaller than the one of the bundle because of the presence of macropores. Compression modulus can be found in the literature or experimentally. For this study, uniaxial compression tests have been performed on dry carbon NCF and the tangent compression modulus was around $0.9 \mathrm{GPa}$ at the fiber volume fraction of $65 \%$. The difference between that experimental value and the estimated values are due to the micromechanical assumptions (no sliding and no rotations) which stiffen the modeled material. Also, concerns remain regarding the way to measure and calculte the compression modulus from an experimental curve.

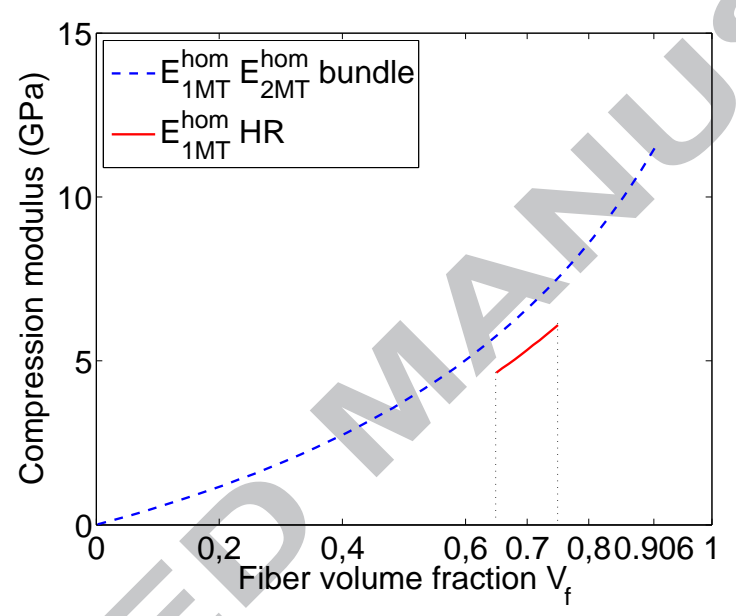

Fig. 10. Calculated compression modulus of the non-crimp UD reinforcement of (high strength) carbon fiber. The transverse Young's modulus of (high strength) carbon fiber is $15 \mathrm{GPa}$

\subsection{Permeability estimate}

As seen previously (section 3.2.2), the estimation schemes are not well adapted to the permeability calculation of the unidirectional assemblies of single-scale porosity because of the difficulty to choose a reference medium that allows to perform calculations. But for the reinforcement of double-scale porosity, the reference medium can be chosen as the bundle of single-scale porosity, whose permeability is calculated by the Gebart's model [49].

The permeabilities will be estimated by the Mori-Tanaka scheme for another non-crimp UD reinforcement made of glass fibers (Fig. 11). It is also a reinforcement containing double-scale porosity, identically to the previous fabric studied, i.e. there are intra-bundle micropores and inter-bundle and inter-layer 
macropores (Fig. 12). The longitudinal and transverse threads somehow hinder the resin flow, thus the pores are assumed ellipsoidal and not cylindrical.

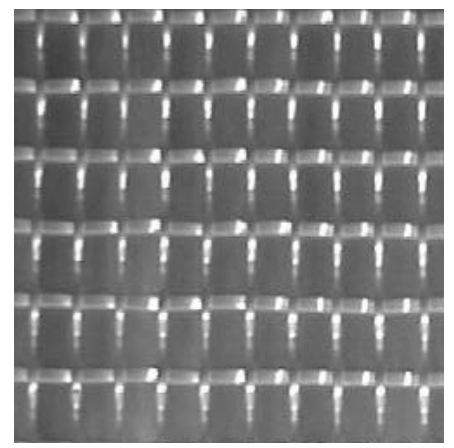

Fig. 11. Glass non-crimp unidirectional reinforcement

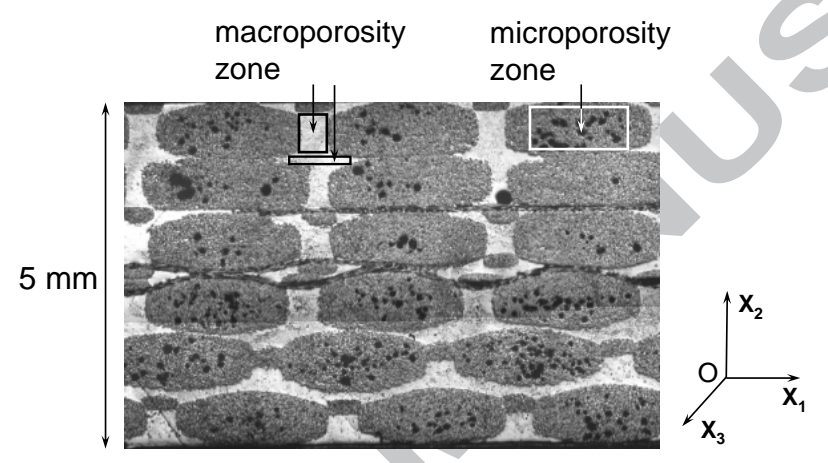

Fig. 12. Transverse micrography of the stacking of 6 layers of glass non-crimp unidirectional reinforcement

The Mori-Tanaka scheme is applied by considering that there are two ellipsoidal macropore families: ellipsoidal inter-bundle macropore and penny-shape inter-layer macropore, which are present in a reference medium, the bundle (Fig. 13).

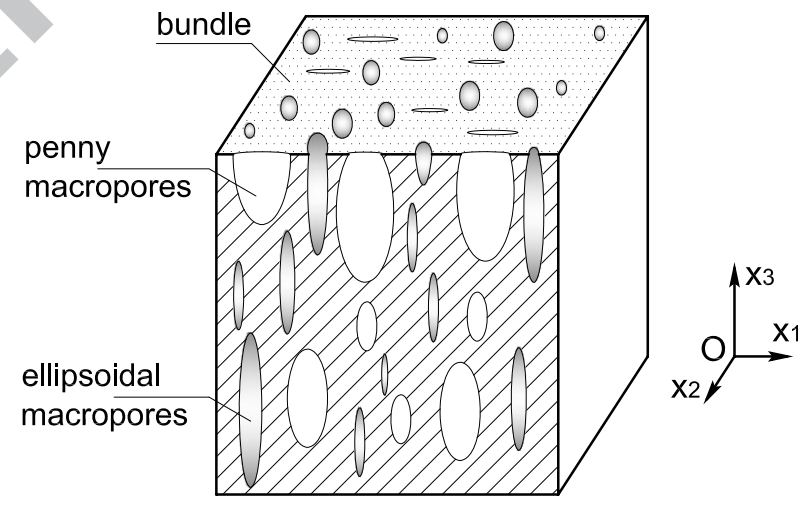

Fig. 13. Modelled glass UD NCF for the Mori-Tanaka scheme

The properties of the ellipsoidal macropore are determined from micrographies and presented in Tab. 2. Some values of ellipsoidal macropores are extrapolated by modifying the semi-axis $a_{2}$ by Eq. 23 for more values of the estimated 
permeability curves. The other semi-axes are unchanged for the different thicknesses of the glass non-crimp reinforcement.

Table 2

Properties of the macropores

\begin{tabular}{|l|c|c|}
\hline & Ellipsoidal macropore & Penny-shape macropore \\
\hline Volume fraction & 0.140 & 0.031 \\
\hline Semi-axis $a_{1}(\mathrm{~mm})$ & 0.175 & 1.077 \\
\hline Semi-axis $a_{2}(\mathrm{~mm})$ & 0.379 & 0.013 \\
\hline Semi-axis $a_{3}(\mathrm{~mm})$ & 1.650 & 1.650 \\
\hline
\end{tabular}

The homogeneous permeability tensor $\boldsymbol{K}_{M T}^{\text {hom }}$ of the glass non-crimp reinforcement is calculated for the material of $n$ pore families [43]

$$
\boldsymbol{K}_{M T}^{h o m}=\boldsymbol{K}_{B}+\left[\sum_{i=1}^{n} f_{i}\left(\boldsymbol{K}_{i}-\boldsymbol{K}_{B}\right) \boldsymbol{D}_{i}\right]\left[\boldsymbol{I}+\sum_{i=1}^{n} f_{i}\left(\boldsymbol{D}_{i}-\boldsymbol{I}\right)\right]^{-1}
$$

where:

$$
\boldsymbol{D}_{i}=\left[\boldsymbol{I}+\boldsymbol{P}_{i}\left(\boldsymbol{K}_{i}-\boldsymbol{K}_{B}\right)\right]^{-1}
$$

where $\boldsymbol{K}_{B}$ is the permeability tensor of the bundle (the reference medium) whose diagonal components are determined by the Gebart's model (Eqs. 20 and 21$), \boldsymbol{K}_{i}$ are the macropore permeabilities, they are calculated as the flow in a tube of elliptical section (the section of the penny-shape and ellipsoidal macropore, Eq. 17), $f_{i}$ is the volume fraction of the $i$ pore family and $P_{i}$ is defined as in Eq. 14. Connectivity is provided by the reference media (permeable bundle) and the presence of macro ellipsoidal pores.

The permeability curves estimated by the Mori-Tanaka scheme for the unidirectional glass non-crimp reinforcement are presented in Fig. 14. The permeability according to the longitudinal direction is compared to experimental values, which have been measured by compression and injection methods [23]. The estimated and experimental results are in good agreement. The permeabilities are lower than the experimental ones. This is probably due to the fact that only ellipsoidal pores have been used in the calculations. Such choice has been made because a small weaving thread in present along the main bundles and hinders the flow. In reality there must be a stronger pore connectivity that should be represented by cylindrical pores which would give a higher estimated permeability. Since the determination of the size and the amount of such cylindrical pore was not possible from micrographs, it has been decided to only consider ellipsoidal pores. Such results emphasize the interest in such 


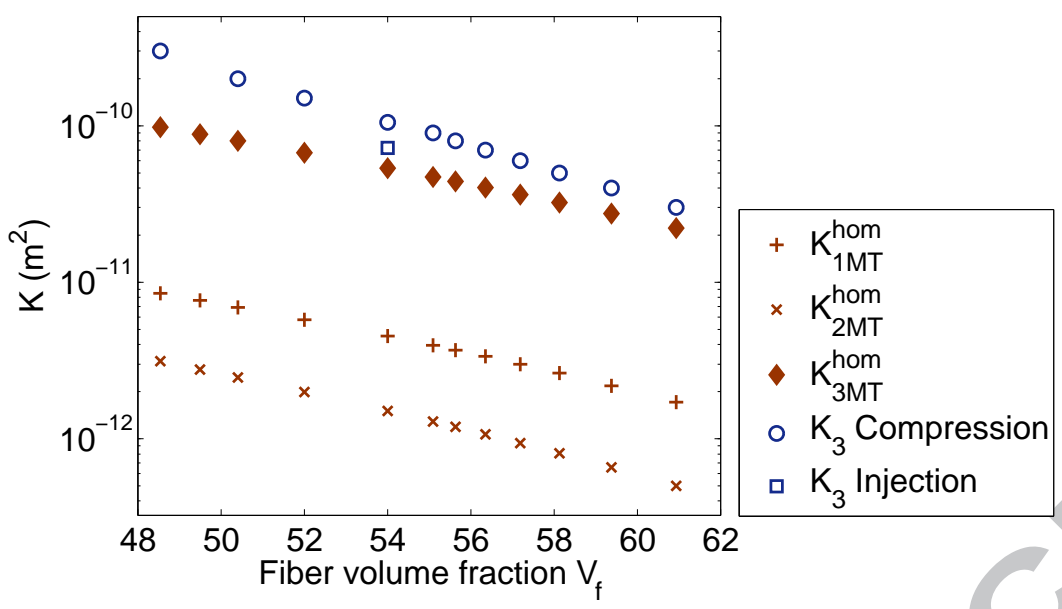

Fig. 14. Estimated permeabilities by the Mori-Tanaka scheme compared to the experimental values for the glass UD NCF

unified method allowing to get hydromechanical, flow and mechanical properties of fiber reinforcement and also because the permeability is difficult to determine experimentally.

\section{Conclusion}

The microporomechanical theory has been applied to unidirectional reinforcements to calculate Biot coefficients, compression modulus and permeability. This is a development based on the previous study of perfectly straight unidirectional fiber assembly [1], while the fiber in the present work is transversely isotropic, in localized contact and the fibrous material consists of double-scale porosity. This development widens the solution to transversely isotropic fibers (carbon or aramid). Furthermore, the calculation is carried out for the doublescale porosity, orthotropic reinforcement, which is closer to industrial materials in the composites processing.

The present results prove that the Biot coefficients of bundles made of transversely isotropic fibers like carbon or aramid are always lower than one, and for the same porosity, the Biot coefficients of the perfectly straight unidirectional fiber assembly are not much different to that in localized contact.

The micromechanical approach stiffens the fibrous material because it hinders the possible sliding and rotations that can occur in such non-cohesive materials. The result validity is therefore limited to high fiber volume fractions. However, using consecutive homogenizations allow to obtain mechanical properties at lower fiber volume fractions.

The permeability tensor is also calculated by the microporomechanical ap- 
proach, but it is not well adapted to the misaligned unidirectional fiber assembly of single-scale porosity because of the difficulty to choose a reference medium. The permeability of an array of solid fiber should be determined with regular fluid mechanics methods. However, permeabilities of the reinforcement of double-scale porosity can be predicted by this continuum approach, and they have showed good agreement with experimental results.

The obtained results prove that the microporomechanical approach could be applied to the fiber reinforcement in the processing of composites and offers an unified consistent framework to model fluid-related and fiber-related phenomena involved in the composites manufacturing processes.

\section{References}

[1] Tran T, Binetruy C, Comas-Cardona S, Abriak NE. Microporomechanical behaviour of perfectly straight unidirectional fiber material: theoretical and experimental. Compos Sci Technol 2009;69:199-206.

[2] Mazumdar SK. Composites manufacturing: materials, product and process engineering. CRC Press, 2001.

[3] Binetruy C, Krawczak P. Compression des renforts dans les procédés LCM. 2: Modélisation de la perméabilité. Rev Compos Mater Av 2002;12:265-84.

[4] Gauvin R, Chibani M. The modeling of mold filling in resin transfer molding. Int Polym Proc 1986;1:42-46.

[5] Kardos JL, Lam RC. The permeability and compressibility of aligned and cross-plied carbon fiber beds during processing of composites. Polym Eng Sci 1991;31:1064-70.

[6] Kenny JM, Molina G, Trivisano A. Mathematical modelling of resin transfer molding of composites for automotive applications. In: ICCM proceedings, Madrid, Spain, July 12-16th, 1993; p. 513-20.

[7] Bruno A, Mazzola M, Molina G. Modélisation de l'écoulement et validation expérimentale du procédé RTM. Composites 1992;3:397-404.

[8] Adams KL, Miller B, Rebenfeld L. Forced in-plane flow of an epoxy resin in fibrous networks. Polym Eng Sci 1986;26:1434-41.

[9] Advani SG, Bruschke MV. A finite element/control volume approach to mold filling in anisotropic porous media. Polym Compos 1990;11:398-405.

[10] Lee LJ, Perry MJ, Wang TJ. Analysis of permeability and void formation in resin transfer molding. In: ANTEC proceedings, Detroit, USA, May 3-7th, 1992; p. $756-60$. 
[11] Endruweit A, Luthy T, Ermanni P. Investigation of the influence of textile compression on the out of plane permeability of a bidirectional glass fiber fabric. Polym Compos 2002;23:538-54.

[12] Bauer S, Boucher D, Gutowski TG. Consolidation experiments for laminate composites. J Compos Mater 1987;21:650-69.

[13] Han KK, Lee CW, Rice BP. Measurements of the permeability of fibre preforms and applications. Compos Sci Technol 2000;60:2435-41.

[14] Ahn SH, Lee WI, Springer GS. Measurement of the three-dimensional permeability of fiber preforms using embedded fiber optic sensors. J Compos Mater 1995;29:714-33.

[15] Weitzenbock JR, Shenoi RA, Wilson PA. Measurements of three-dimensional permeability. Composites Part A 1998;29:159-69.

[16] Woerdeman DL, Phelan FR, Parnas RS. Interpretation of 3D permeability measurements for RTM modelling. Polym Compos 1995;16:470-80.

[17] Lundstrom TS, Stenberg R, Bergstrom R, Partanen H, Birkeland PA. In-plane permeability measurements: a nordic round-robin study. Composites Part A 2000;31:29-43.

[18] Parnas RS, Flynn KM, Dal-Favero ME. A permeability database for composites manufacturing. Polym Compos 1997;18:623-33.

[19] Feser JP, Prasad AK, Advani SG. Experimental characterization of in-plane permeability of gas diffusion layers. J Power Sources 2006;162:1226-31.

[20] Pomeroy R, Grove S, Summerscales J, Wang Y, Harper A. Measurement of permeability of continuous filament mat glass-fibre reinforcements by saturated radial airflow. Composites Part A 2007;38:1439-43.

[21] Ait Si Ahmad M, Fasi-Ferhi O, Poitou A, Bojji C. Model for determining the permeability variation of fiber mat reinforcement submitted to compression. Mec Ind 2002;3:27-34.

[22] Buntain MJ, Bickerton S. Compression flow permeability measurement: a continuous technique. Composites Part A 2003;34:445-57.

[23] Comas-Cardona S, Binetruy C, Krawczak P. Unidirectional compression of fibre reinforcements. Part 2: A continuous permeability tensor measurement. Compos Sci Technol 2007;67:638-45.

[24] Lundstrom TS. The permeability of non-crimp stitched fabrics. Composites Part A 2000;31:1345-53.

[25] Simacek P, Karbhari VM. Notes on the modeling of preform compaction. I: Micromechanics at the fiber bundle level. J Reinf Plast Compos 1996;15:86122. 
[26] Song YS, Chung K, Kang TJ, Youn JR. Prediction of permeability tensor for three dimensional circular braided preform by applying a finite volume method to a unit cell. Compos Sci Technol 2004;64:1629-36.

[27] Fournier R, Coupez T, Vincent M. Numerical determination of the permeability of fibre reinforcement for the RTM process. Revue Européenne des Eléments Finis 2005;14:803-18.

[28] Belov EB, Lomov SV, Verpoest I, Peters T, Roose D, Parnas RS, Hoes K, Sol H. Modelling of permeability of textile reinforcements: lattice Boltzmann method. Compos Sci Technol 2004;64:1069-80.

[29] Comas-Cardona S, Groenenboom PHL, Binetruy C, Krawczak P. Simulation of liquid molding processes using a generic mixed FE-SPH method. Revue Européenne des Eléments Finis 2005;14:867-83.

[30] Nedanov PB, Advani SG. Numerical computation of the fiber preform permeability tensor by the homogenization method. Polym Compos 2002;23:758-70.

[31] Chen B, Cheng AHD, Chou TW. A nonlinear compaction model for fibrous preforms. Composites Part A 2001;32:701-07.

[32] Toll S, Manson JAE. An analysis of the compressibility of fiber assemblies. 6th International Conference on Fiber Reinforced Composites, Institute of Materials. Newcastle-upon-Tyne, UK, 1994; p. 25/1-25/10.

[33] Lekakou C, Johari MAKB, Bader MG. Compressibility and flow permeability of tow-dimensional woven reinforcements in the processing of composites. Polym Compos 1996;17:666-72.

[34] Melro AR, Camanho PP, Pinho ST. Generation of random distribution of fibres in long-fibre reinforced composites. Compos Sci Technol 2008;68:2092-102.

[35] Pyrz R. Quantitative description of the microstructure of composites. Part I: Morphology of unidirectional composite systems. Compos Sci Technol 1994;50:197-208.

[36] Pyrz R. The application of morphological methods to composite materials. Comprehensive Composite Materials 2003;2:553-76.

[37] Chiu SN. Aboav-Weaire's and Lewis's laws: a review. Mater Charact 1995;34:149-65.

[38] Soille P. On the validity of fractal dimension measurements in image analysis. $\mathrm{J}$ Vis Commun Image R 1996;7:217-29.

[39] Gusev AA, Hine PJ, Ward IM. Fiber packing and elastic properties of a transversely random unidirectional glass/epoxy composite. Compos Sci Technol 2000;60:535-41.

[40] Torquato S, Lu B. Chord-length distribution for 2-phase random-media. Physics Review E 1993;47:2950-53. 
[41] Yurgartis SW. Measurement of small angle fiber misalignments in continuous fiber composites. Compos Sci Technol 1987;30:279-93.

[42] Lee J, Soutis C. Thickness effect on the compressive strength of T800/924C carbon fiber-epoxy laminates. Composites Part A 2005;36:213-27.

[43] Dormieux L, Kondo D, Ulm FJ. Microporomechanics. John Wiley \& Sons Inc.; 2006.

[44] Eshelby JD. The determination of the elastic field of an ellipsoidal inclusion, and related problems. Proc Roy Soc Lond A 1957;241:376-96.

[45] Walpole LJ. Fourth-rank tensors of the thirty-two crystal classes: multiplication tables. Proc R Soc Lond A 1984;391:149-79.

[46] Camacho CW, Tucker CL, Yalvac S, Mcgee RL. Stiffness and thermal expansion predictions for hybrid short fiber composites. Polym Composite 1990;11:229-39.

[47] Chamis CC. Simplified composite micromechanics for predicting microstresses. J Reinf Plast Compos 1987;6:268-89.

[48] Comolet R. Mécanique expérimentale des fluides. Tome 2: Dynamique des fluides réels, turbomachines. Masson; 1994.

[49] Gebart BR. Permeability of unidirectional reinforcements for RTM. J Compos Mater 1992;26:1100-33.

[50] Dormieux L, Kondo D. Approche micromécanique du couplage perméabilitéendommagement. Compte Rendu Mécanique 2004;332:135-40. 